\title{
Ocorrência de oocistos de protozoários gastrointestinais em Passeriformes, Psitaciformes e Galiformes comercializadas em pet shops de Salinas, Minas Gerais
}

\author{
Geraldo Rodrigues Gomes Neto*1; Maria Tereza Ribeiro Silva Nogueira ${ }^{2}$; Isabela Aquino Pereira ${ }^{3}$; Vanessa \\ Paulino da Cruz Vieira ${ }^{4}$
}

DOI: https://doi.org/10.35699/2447-6218.2021.35359

\begin{abstract}
Resumo
As parasitoses gastrointestinais, incluindo as causadas por protozoários coccídios, são doenças que podem causar alterações subclínicas ou mesmo morte de aves submetidas ao estresse, mantidas em gaiolas de alta densidade populacional. Assim, objetivou-se detectar a presença de oocistos de protozoários gastrointestinais em amostras fecais oriundas de recintos de aves das ordens Passeriformes, Psitaciformes e Galiformes comercializadas em pet shops de Salinas, Minas Gerais, Brasil. Amostras de fezes foram coletadas de 15 recintos de três espécies de Psittaciformes, duas espécies de Galiformes e uma espécie de Passeriformes. Dentre os recintos avaliados, haviam Passeriformes da espécie Serinus canaria (canário-belga), Psitaciformes das espécies Nymphicus hollandicus (calopsita), Melopsittacus undulates (periquito-australiano) e Agapornis personatus (Agapornis) e Galiformes das espécies Coturnix Coturnix japonica (codorna japonesa) e Gallus gallus domesticus (galinha doméstica). As amostras foram processadas usando a técnica de flutuação com solução saturada de cloreto de sódio (Willis-Mollay) e as amostras positivas para oocistos de protozoários coccídios foram submetidas à esporulação em dicromato de potássio $1,0 \%$ à temperatura ambiente. Ovos de helmintos não foram detectados, entretanto oocistos de protozoários foram observados em dois recintos de codorna japonesa e um recinto de canário-belga. O presente trabalho expõe a presença de coccídios de aves comercializadas no município de Salinas, no estado de Minas Gerais. O conhecimento parasitológico obtido pode contribuir para a decisão de medidas preventivas, visando a melhoria da saúde e bem-estar das aves comercializadas.
\end{abstract}

Palavras-chave:. Aves exóticas. Coprologia. Endoparasitos. Saúde animal.

\section{Occurrence of gastrointestinal protozoan oocysts in Passeriformes, Psittaciformes and Galliformes sold in pet shops in Salinas, Minas Gerais}

\begin{abstract}
Gastrointestinal parasitoses, including those caused by coccidial protozoa, are diseases that can cause subclinical changes or even death in birds submitted to stress, kept in cages with high population density. Thus, we aimed to detect the presence of gastrointestinal protozoan oocysts in fecal samples from bird enclosures of the orders Passeriformes, Psittaciformes and Galiformes sold in pet shops in Salinas, Minas Gerais, Brazil. Fecal samples were collected from 15 enclosures of three Psittaciformes species, two Galliformes species and one Passeriformes species. Among the enclosures evaluated were Passeriformes of the species Serinus canaria (canary), Psittaciformes of the species Nymphicus hollandicus (cockatiel), Melopsittacus undulates (budgerigar) and Agapornis personatus (lovebird) and Galiformes of the species Coturnix coturnix japonica (japanese quail) and Gallus gallus domesticus (domestic chicken). Samples
\end{abstract}

${ }^{1}$ Instituto Federal de Educação, Ciência e Tecnologia do Norte de Minas Gerais. Salinas, MG. Brasil. https://orcid.org/0000-0002-2903-957

${ }^{2}$ Instituto Federal de Educação, Ciência e Tecnologia do Norte de Minas Gerais. Salinas, MG. Brasil. https://orcid.org/0000-0003-2965-5812

${ }^{3}$ Instituto Federal de Educação, Ciência e Tecnologia do Norte de Minas Gerais. Salinas, MG. Brasil. https://orcid.org/0000-0002-3671-3104

${ }^{4}$ Instituto Federal de Educação, Ciência e Tecnologia do Norte de Minas Gerais. Salinas, MG. Brasil. https://orcid.org/0000-0003-2276-2623

*Autor para correspondência: geraldorodrigues179@gmail.com

Recebido para publicação em 23 de Julho de 2021. Aceito para publicação 30 de Setembro de 2021. e-ISSN: 2447-6218 / ISSN: 2447-6218. Atribuição CC BY. 
were processed using the sodium chloride saturated solution floatage technique (Willis-Mollay) and samples positive for coccidial protozoan oocysts were subjected to sporulation in 1.0\% potassium dichromate at room temperature. Helminth eggs were not detected, but oocysts of protozoa were observed in two enclosures of Japanese quail and one enclosure of canary. The present work exposes the presence of coccidia in birds commercialized in the municipality of Salinas, in the state of Minas Gerais. The parasitological knowledge obtained can contribute to the decision of preventive measures, aiming at improving the health and welfare of commercialized birds.

Keywords: Animal health. Coprology. Endoparasites. Exotic birds.

\section{Introdução}

A utilização de aves como pets é uma prática extensamente difundida, principalmente as espécies dóceis, atraentes e de fácil manejo (Reis, 2015). O conhecimento das doenças que acometem as aves que permanecem mantidas em cativeiro se faz importante, já que técnicas adequadas de manejo, em conjunto com o tratamento adequado, podem contribuir de forma positiva, para a manutenção e o restabelecimento da saúde desses animais (Ayres et al., 2016).

As doenças parasitárias estão entre os principais problemas de saúde que afetam as aves. Tais doenças podem causar alterações subclínicas ou mesmo morte de aves submetidas ao estresse, quando mantidas em gaiolas de alta densidade populacional. Embora pouco se saiba sobre as taxas de infecções parasitárias de aves em diferentes regiões do Brasil, sabe-se que as coccidioses são prevalentes em aves em cativeiro. As aves podem ser parasitadas por diversos gêneros, com destaque para Eimeria sp. e Isospora sp., que apresentam geralmente ciclo de vida intestinal, mas também podem apresentar o ciclo extra intestinal (Berto et al., 2014).

Aves parasitadas por Eimeria spp. podem reduzir o desempenho no crescimento através do comprometimento da função intestinal (Kim et al., 2017; Lu et al., 2019). Já na infecção por Isospora spp., os sinais clínicos incluem apatia, redução do apetite e penas arrepiadas, o que resulta em alta mortalidade (Oliveira et al., 2017).

Aves de todas as idades podem ser acometidas por esses parasitos, que causam importantes modificações na estrutura das vilosidades intestinais, acarretando a diminuição da absorção de nutrientes, podendo causar diarreias sanguinolentas ou não, ocasionando prejuízos econômicos em pequenas e grandes criações (Balthazar, 2011). As aves mais frequentemente parasitadas por este grupo são algumas espécies de Passeriformes, Psitaciformes, Falconiformes, Galiformes e Columbiformes (Samour, 2010).

Os oocistos são as estruturas de resistência dos coccídios pois protegem os esporozoítos, que são as formas infectantes. Geralmente são liberados junto com as fezes do hospedeiro e por esta razão são utilizados para diagnóstico, descrições e estudos sistemáticos desde os primórdios da parasitologia (Berto et al., 2014).
Os exames coproparasitológicos apresentam baixo custo, possuem importância clínica, indicam a população de endoparasitos e os tratamentos terapêuticos mais adequados, sendo a técnica de flutuação a preferida, pela menor presença de sujidades, sendo possível fazer uma melhor visualização dos oocistos. Nessas técnicas são utilizadas soluções saturadas com elevada densidade promovendo a flutuação dos oocistos presentes nas fezes, fazendo com que eles permaneçam na superfície do sobrenadante. Entre elas está a Técnica de Willis - Mollay (Flutuação e solução salina): esta é muito utilizada para ver se há ou não presença de oocistos de protozoários (Sousa et al., 2020). Adicionalmente, a utilização de compostos químicos ricos em oxigênio tais como o dicromato de potássio (K2Cr2O7) 1\%, estimulam a esporulação dos oocistos em laboratório (Bowman, 2010).

Sabe-se que a criação de aves tem aumentado significativamente e pouco se analisa sobre os parasitos que as acometem. Diante disso, objetivou-se com este trabalho detectar a presença de oocistos de protozoários gastrointestinais em amostras de fezes de aves das ordens Passeriformes, Psitaciformes e Galiformes comercializadas em pet shops de Salinas, Minas Gerais.

\section{Material e métodos}

\section{Coleta das amostras}

Em outubro de 2019, foram coletadas amostras fecais de quinze recintos de aves das ordens Psittaciformes, Galiformes e Passeriformes. As coletas foram realizadas em três pet shops localizados no centro do município de Salinas, norte de Minas Gerais.

Dentre os recintos avaliados, cinco continham Passeriformes da espécie Serinus canaria (canário-belga). Seis recintos continham Psitaciformes, onde três pertenciam a espécie Nymphicus hollandicus (calopsita), dois a espécie Melopsittacus undulates (periquito-australiano) e um a Agapornis personatus (Agapornis). E, os quatro recintos restantes, continham Galiformes, com dois da espécie Coturnix Coturnix japonica (codorna japonesa) e dois da espécie Gallus gallus domesticus (galinha doméstica).

Foram coletadas uma amostra de cada recinto (gaiolas ou viveiros) depositadas nas bandejas inferiores 
Ocorrência de oocistos de protozoários gastrointestinais em Passeriformes, Psitaciformes e Galiformes comercializadas em pet shops de Salinas, Minas Gerais

dos mesmos (Figura 1), com auxílio de uma espátula,

sendo armazendas em recipientes de plástico limpos e identificados, totalizando 15 amostras fecais analisadas.

Figura 1 - Coleta de amostras de fezes depositadas na bandeja inferior do recinto de indivíduos da espécie Serinus canaria comercializados em pet shop de Salinas, Minas Gerais. (Fonte: arquivo da pesquisa).

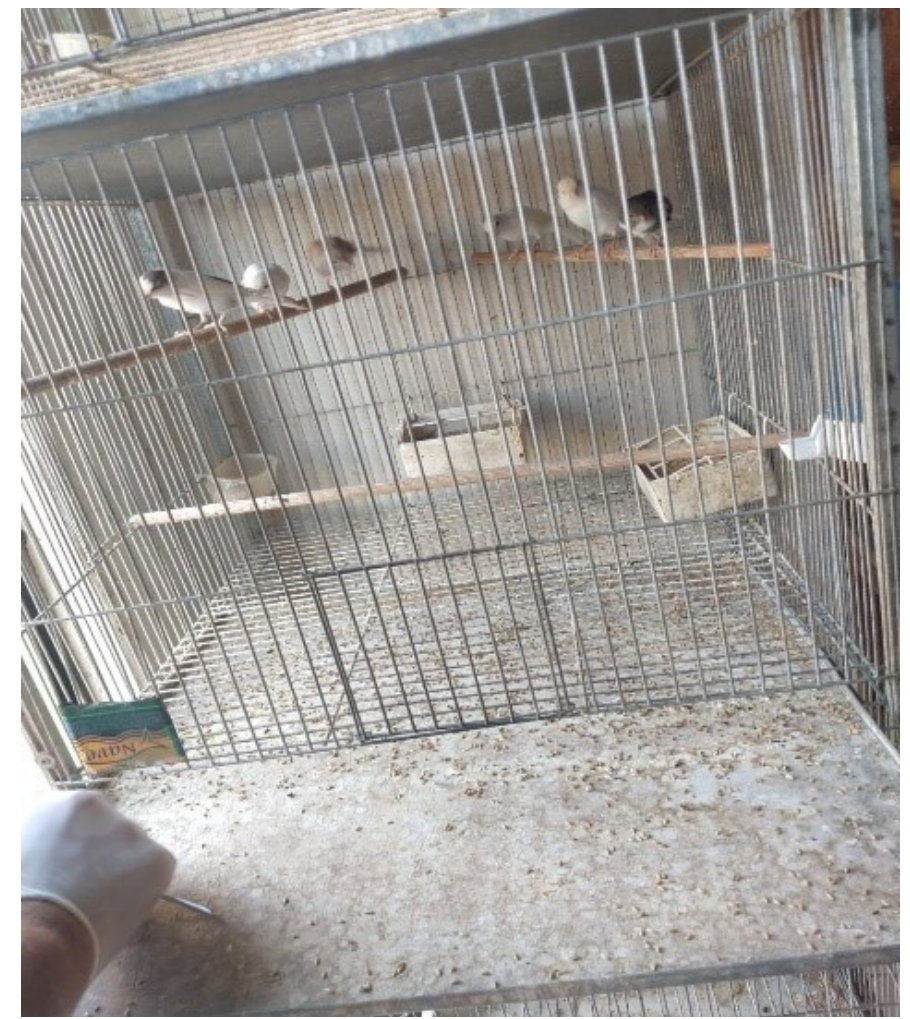

\section{Processamento das amostras}

As amostras foram acondicionadas em caixa térmica e encaminhadas ao laboratório, mantidas em refrigeração à $8{ }^{\circ} \mathrm{C}$ e processadas em até 24 horas. $\mathrm{O}$ processamento e análise das amostras foram realizados no Laboratório de Parasitologia Veterinária (LPV) do Instituto Federal do Norte de Minas Gerais, Campus Salinas. As amostras foram processadas pela técnica de flutuação com solução saturada de cloreto de sódio (Willis-Mollay) descritos por Hendrix e Robinson (2014).

As amostras positivas para oocistos de protozoários coccídios foram colocadas em placas de Petri com solução de dicromato de potássio a 1\% (K2Cr2O7) para esporulação à temperatura ambiente, para diferenciá-los entre os gêneros Isospora ou Eimeria de acordo com a descrição de Bowman (2010). Para visualização e identificação dos oocistos, as lâminas foram submetidas à microscopia óptica, nas objetivas de 10x e 40x.

\section{Resultados e discussão}

Não foi detectada a presença de ovos e/ou larvas de helmintos, entretanto na Tabela 1 podem ser observados os resultados referentes à presença de oocistos de protozoários coccídios em amostras de dois recintos de codorna japonesa (Fig. 2) e em um recinto de canário-belga (Fig. 3).
O fato da presença de oocistos de coccídios ter sido detectada em três dos 15 recintos estudados, pode ser explicado pelo fato dos recintos que eram suspensos, onde as aves não tinham acesso direto às fezes e seus comedouros e bebedouros estavam isolados dos locais contaminados. Cabe ressaltar também que esses recintos não estavam acessíveis à animais externos, nos quais as chances de infecção parasitária são maiores, já que eles vivem em um ambiente aberto em que a contaminação se manifesta na forma pouco higiênica dos hábitos alimentares e por não serem submetidos à tratamentos antiparasitários.

Na região Sudeste, outros trabalhos envolvendo o levantamento do parasitismos em aves de cativeiro. Dentre eles, Vasconcelos et al. (2012), relataram óbito devido ao intenso parasitismo por coccídios em dois bicudos (Oryzoborus maximiliani) atendidos no setor de animais selvagens do Departamento de Patologia Clínica Veterinária da Universidade Federal Fluminense.

Ainda, ao avaliar os parasitos em pássaros silvestres e exóticos de criatórios particulares no município de Alegre, ES, Carneiro et al. (2011) observaram que $50 \%$ das amostras coletadas estavam positivas para a presença de oocistos de coccídios. Já Silva et al. (2014) detectaram oocistos de coccídeos em fezes de bicudos criados em cativeiro, relatando não terem encontrado sintomatologia clínica aparente nas aves. 
Tabela 1 - Presença de oocistos de protozoários coccídios observados na técnica coproparasitológica de flutuação com solução saturada de cloreto de sódio (Willis-Mollay), de acordo com Hendrix e Robinson (2014), em espécies das ordens Passeriformes, Psittaciformes e Galiformes comercializadas em pet shops de Salinas, Minas Gerais, Brasil, no mês de outubro de 2019.

\begin{tabular}{lcc}
\hline \multicolumn{2}{c}{ Oocistos de protozoários coccídios em amostras fecais de recintos de aves } \\
\hline Espécie & Recinto & Presença de oocistos \\
\hline Serinus canaria & Ordem Passeriformes & Negativo \\
Serinus canaria & 1 & Negativo \\
Serinus canaria & 3 & Negativo \\
Serinus canaria & 4 & Negativo \\
Serinus canaria & 5 & Positivo7 \\
\hline & Ordem Galiformes & \\
Cortunix cortunix japonica & 6 & Positivo \\
Cortunix cortunix japonica & 7 & Positivo \\
Gallus gallus domesticus & 8 & Negativo \\
Gallus gallus domesticus & 9 & Negativo \\
\hline & 10 & \\
Nymphicus hollandicus & Ordem Psittaciformes & Negativo \\
Nymphicus hollandicus & 10 & Negativo \\
Nymphicus hollandicus & 12 & Negativo \\
Melopsttacus undulates & 13 & Negativo \\
Melopsttacus undulates & 14 & Negativo \\
Agapornis personatus & Negativo \\
\hline
\end{tabular}

Figura 2 - Oocistos de protozoários coccídios observados através da técnica de flutuação com solução saturada de cloreto de sódio (Willis-Mollay), de acordo com Hendrix e Robinson (2014), nas fezes de indivíduo da espécie Coturnix Coturnix japonica (Objetiva 10X), comercializados em pet shop de Salinas, Minas Gerais. (Fonte: arquivo da pesquisa).

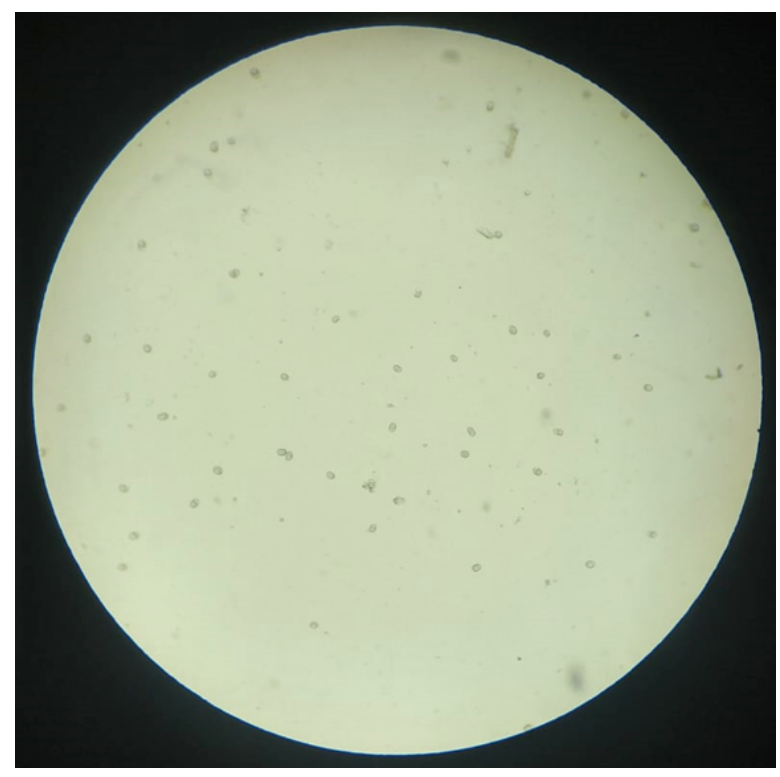


Figura 3 - Oocistos de coccídios observados através da técnica de flutuação com solução saturada de cloreto de sódio (Willis-Mollay), de acordo com Hendrix e Robinson (2014), nas fezes de indivíduo da espécie Serinus canaria (Objetiva 40X), comercializados em pet shop de Salinas, Minas Gerais. (Fonte: arquivo da pesquisa).

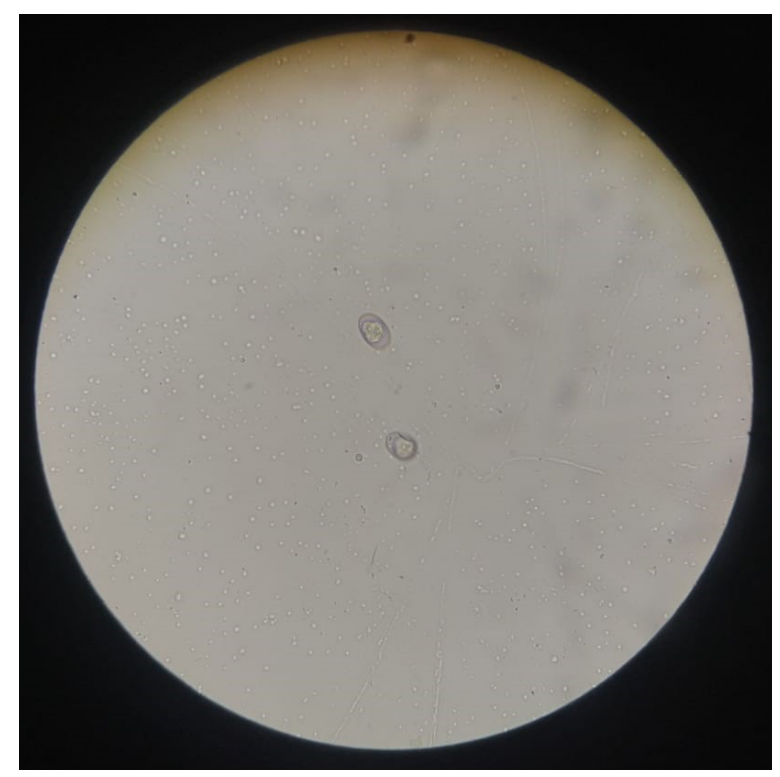

Na região Sul, Zardo et al. (2014), realizando um levantamento das espécies de aves mais encontradas em cativeiro no bairro Camobi, município de Santa Maria - RS, detectaram a presença de coccídios em 15 das 49 amostras de fezes analisadas através da técnica de Willis-Mollay modificada. Os autores supõem que se uma ave está infectada por determinado parasito, as demais aves presentes no mesmo recinto provavelmente também estarão. Adicionalmente, Boll et al., (2017) também relataram a presença de coccídios em fezes de passeriformes alojados no centro de triagem do zoológico de Sapucaia do Sul, Rio Grande do Sul.

Lopes et al. (2021), na região Norte, ao realizar a identificação qualitativa dos endoparasitos que acometem codornas de duas granjas na cidade de Porto Velho - RO, através do método de Willis-Mollay, encontrou oocistos de coccídios esporulados e não esporulados.

Os resultados encontrados são de relevância, uma vez que, de acordo com Balthazar (2011), as coccidioses, que podem ser causadas por protozoários dos gêneros Eimeria e Isospora, vêm a ser direta ou indiretamente responsável por mais da metade dos problemas de saúde das aves de gaiola.

A realização da técnica de esporulação através da adição de solução com dicromato de potássio a $1 \%$ (K2Cr2O7) nas amostras fecais positivas para oocistos foi realizada no intuito de identificar se o parasitismo era em decorrência da infecção por Eimeria ou Isospora. No entanto, não ocorreu a esporulação em nenhuma das três amostras fecais positivas submetidas a essa técnica, o que pode ter sido causado por condições climáticas adversas no período em que as fezes permaneceram nos recintos, tendo possivelmente inviabilizado a fertilidade dos oocistos, tornando-os inférteis ou prejudicando a esporogonia, considerando que os oocistos precisam de condições ideais de umidade, temperatura e oxigenação para que ocorra a esporulação. Em estudo realizado por Toledo et al. (2011) foi observado que a esporulação de oocistos em camas que possuem umidade elevada é maior. As fezes contidas nas bandejas inferiores dos recintos das aves do presente estudo não eram recolhidas diariamente, havendo fezes recém expelidas, com aspecto úmido e brilhante, juntamente com fezes ressecadas.

Segundo Carneiro et al. (2011) em aves exóticas e silvestres a prevenção de parasitoses está relacionada aos cuidados gerais de higiene na criação, alimentação adequada, água de boa qualidade, regras de manejo instituídos corretamente ao tipo de criação, treinamento dos tratadores nos cuidados com o manuseio das aves doentes, exames periódicos de fezes nas aves do plantel.

Mesmo sem ter sido possível determinar por quais espécies de coccídios as aves estavam parasitadas, é possível promover o direcionamento à um tratamento mais específico, uma vez que a terapêutica indicada é a mesma para ambas as espécies de protozoário, evitando-se o uso indiscriminado de medicamentos antiparasitários, sem diagnóstico prévio.

Os resultados obtidos com a realização do presente trabalho, tem sua relevância balizada em sua função de alertar os comerciantes e criadores desses animais, para os riscos aos quais os animais estão expostos, permitindo o controle e profilaxia pertinenetes. É sabido que as coccidioses estão presentes em grande parte das aves, no entanto, este trabalho se faz importante, já que é a primeira vez que é realizado um levantamento como este na cidade de Salinas, Norte de Minas Gerais. 
Gomes Neto, G. R. et al.

Sugere-se a realização de mais estudos nesse âmbito, não unicamente em animais de cativeiro, mas também em granjas, criações extensivas e em aves de vida livre, no intuito de que o conhecimento do parasitismo por protozoários, como neste caso, possa fomentar o planejamento e adoção de medidas de controle e prevenção mais efetivas, visando o bem-estar e a qualidade de vida dessas aves.

\section{Conclusão}

Conclui-se então, que as espécies Serinus canaria da ordem dos Passeriformes e Coturnix Coturnix japonica da ordem dos Galiformes comercializadas em pet shops da cidade de Salinas apresentaram parasitismo por protozoários coccídios.

\section{Referências}

Ayres, M. C. C.; Peixoto, M. S. R.; Silva, W. B.; Gomes, D. M.; Nunes, O. C.; Borges, K. B.; Morais Neto, M. A.; Almeida, M. A. O. 2016. Ocorrência de parasitos gastrintestinais em Psitacídeos, mantidos em Parques Ecológicos na região metropolitana de Salvador, Bahia. Revista Brasileira de Medicina Veterinária, 32: 33-136.

Balthazar, L. M. C. 2011. Diagnóstico das espécies do gênero Isospora Schneider, 1881 (Apicomplexa: Eimeriidae) em pássaros de gaiola. Seropédica: Universidade Federal Rural do Rio de Janeiro, 62f. Dissertação mestrado. Disponível em: https://tede.ufrrj.br/jspui/ handle/jspui/4031.

Berto, B. P.; Mcintosh d.; Lopes, C. W. G. 2014. Studies on coccidian oocysts (Apicomplexa: Eucoccidiococcídiorida). Brazilian Journal of Veterinary Parasitology, 23: 1-15. Doi: http://dx.doi.org/10.1590/ S1984-29612014001.

Boll, A. S.; Marques, S. M. T.; Alievi, M. M. 2017. Parasitas em Passeriformes e Psittaciformes alojados em centro de triagem no Zoológico em Sapucaia do Sul, Rio Grande do Sul, Brasil. Revista Portuguesa de Ciências Veterinárias, 112: 28-34.

Bowman, D. D. 2010. Georgis Parasitologia veterinária. 9. ed. Rio de Janeiro: Saunders Elsevier, $282 \mathrm{p}$

Carneiro, M. B.; Calais júnior, A.; Martins, I. V. F. 2011. Avaliação coproparasitológica e clínica de aves silvestres e exóticas mantidas em criatórios particulares no município de Alegre -ES. Ciência Animal Brasileira, 12: 525-529. Doi: http://dx.doi.org/10.5216/cab.v12i3.6821.

Hendrix, C. M.; Robinson, E. D. 2014. Diagnostic parasitology for veterinary technicians. 4 ed. Elsevier Health Sciences.

Kim, E.; Leung H.; Akhtar N.; Li J.; Barta JR.; Wang Y.; Kiarie E. 2017. Growth performance and gastrointestinal responses of broiler chickens fed corn-soybean meal diet without or with exogenous epidermal growth factor upon challenge with Eimeria. Poultry Science, 96: 3676-3686. Doi: https://doi.org/10.3382/ps/pex192.

Lopes, T. V.; Maia, A. S.; Araújo, T. L. O.; Souza, J. G. S. G.; Schons, S. V.S; Souza, F. A. 2021. Prevalência de endoparasitas em codornas japonesas (coturnix coturnix japonica) em dois aviários comerciais na região amazônica. Research, Society And Development, 10: 1-6. Doi: http://dx.doi.org/10.33448/rsd-v10i9.17967.
Lu, Z.; Thanabalan, A.; Leung, H.; Kakhki, R. A. M; Patterson, R.; Kiarie, E. G. 2019. The effects of feeding yeast bioactives to broiler breeders and/or their offspring on growth performance, gut development, and immune function in broiler chickens challenged with Eimeria. Poultry Science, 98: 6411-6421. Doi: https://doi.org/10.3382/ps/pez479.

Oliveira, P. S.; Ferreira, M. A.; Silva, L. M.; Rodrigues, M. B.; Cardozo, S.V.; Berto, B. P. 2017. Diversity and distribution of coccidia of wild birds in an Atlantic forest fragment area in southeastern Brazil. Revista Brasileira de Parasitologia Veterinária, 26: 457-464. Doi: http://dx.doi. org/10.1590/s1984-29612017063.

Reis, E. J. C. 2015. Leveduras patogênicas em fezes de aves de cativeiro: relação fenotípica e genotípica entre os isolados. São José do Rio Preto: Universidade Estadual Paulista "Júlio de Mesquita Filho", 100f. Dissertação mestrado. Disponível em: http://hdl.handle. net/11449/136688.

Samour, J. 2010. Medicina aviária. 2 ed. Espanha: Elsevier.

Silva, D. C.; Homem, C. G.; Alex, A. N.; Silva, V. C.; Meireles, M. V. 2014. Pesquisa de Oocistos de Isospora spp. em Passeriformes Criados em Cativeiro. Ciência Animal Brasileira, 15: 484-489. Doi: https:// doi.org/10.1590/1089-6891v15i422558.

Sousa, M. C.; Vial, J. R.; Teixeira, R. H. F; Nakaghi, A. C. H. 2020. Comparação de testes coproparasitológicos em criadouro comercial de psitacídeos exóticos. Revista Agrária Acadêmica, 3: 100-107. Doi: doi. org/10.32406/v3n5/2020/100-107/agrariacad.

Toledo, G. A.; Almeida, J. D. M.; Almeida, K. S.; Freitas, F. L. C. 2011. Coccidiose em frangos de corte criados na região de Araguaína, Estado do Tocantins, Brasil. Revista Brasileira de Parasitologia Veterinária, 20: 249-252. Doi: https://doi.org/10.1590/S1984-29612011000300014.

Vasconcelos, T. C. B.; Longa, C. S.; Campos, S. D. E. 2012. Coccidiose em Sporophila maximiliani (Passeriformes: emberizidae): Relato de dois casos. Revista Brasileira de Medicina Veterinária, 34: 261-264.

Zardo, E. L.; Behr, E. R.; Macedo, A.; Pereira, L. Q.; Lovato. 2014. Aves nativas e exóticas mantidas como animais de estimação em Santa Maria, RS, Brasil. Revista Acta Ambiental Catarinense, 11:33-42. 